Maria Helena Ruzany 1

Carla Lourenço Tavares Andrade 2

Maria Angel a Pires Esteves 2

Maria de Fátima Pina 2

Célia Landman Szwarcwald 2

\section{Avaliação das condições de atendimento do Programa de Saúde do Adolescente no Município do Rio de Janeiro}

\author{
Evaluation of operational conditions \\ in the Adolescent Health Care Program, \\ Rio de Janeiro
}

\footnotetext{
1 Núcleo de Estudos da Saúde do Adolescente Av. 28 de Setembro 109 (fundos), Pavilhão Floriano Stoffel, Rio de Janeiro, RJ 20551-030, Brasil. mruzany@attglobal.net 2 Departamento de Informações em Saúde, Centro de Informação Científica e Tecnológica, Fundação Oswaldo Cruz. Av. Brasil 4365, Rio de Janeiro, RJ 21045-900, Brasil.
}

\begin{abstract}
This study focuses on conditions in the public health centers providing comprehensive care to the adolescent population in the city of Rio de Janeiro. A structured questionnaire was administered to the coordinators of 70 public health centers (70/78). Based on the data, the public health centers were stratified according to basic conditions for providing full care to adolescents. The authors developed a spatial study of the demographic concentration and main problems, producing thematic maps. Of the 49 public heal th centers that participate in the Adolescent Health Program, 12\% were classified in "good" condition and $45 \%$ were consi dered "fair". Among the administrative regions with the highest concentration of adolescents, only six (6/17) were in good or fair condition. The authors conclude that to increase the effectiveness of the city's Adol escent Health Program it is necessary to improve the working conditions of their health staff, taking local health problems, the concentration of adolescents, and their demands for services into account.
\end{abstract}

Key words Adolescence; Adolescent Health Services; Spatial Analysis; Evaluation Studies

Resumo A pesquisa consistiu no estudo das condições básicas para a prestação da atenção integral aos adolescentes nas Unidades de Saúde (US) do Município do Rio de Janeiro. Inicial mente aplicou-se um questionário estruturado aos coordenadores de 70 centros e postos de saúde (70/ 78). Em segui da, com os resul tados obti dos, criou-se um índi ce que sintetizava as condições bási cas ofereci das para a prestação do atendimento e procedeu-se a estratificação da rede pública ambulatorial. O estudo espacial da concentração da população adolescente e de seus principais problemas deu origem a três mapas temáticos. Entre as 70 US estudadas, 49 partici pavam do Programa de Saúde do Adol escente (PROSAD). Destas, $12 \%$ foram classificadas como em "boas" condições e 45\% como "regul ares". O estudo espacial demonstrou que somente seis, das 17 US si tuadas nas áreas de mai or densi dade demográfica, estavam em condi ções boas ou regulares. Concluiu-se que, para aumentar a efetividade do PROSAD do Município do Rio de Janeiro, devese mel horar as condi ções dos profissi onais para prestar atenção integral a este grupo etário, levando em consi deração os problemas de saúde em nível local, a densidade demográfica da população adolescente por área geográfica ea demanda de serviços.

Palavras-chave Adolescência; Serviços de Saúde à Adolescentes; Análise Espacial; Estudos de Avaliação 


\section{Introdução}

A saúde do adolescente em nível internacional vem recebendo atenção especial desde o início do século $X X$, quando profissionais de saúde observaram que este grupo populacional merecia estudos aprofundados pelas suas particularidades (Gates, 1918; Hall, 1904). Desde então, profissionais de saúde de alguns serviços, em diferentes países, passaram a desenvolver serviços específicos de atenção à saúde deste grupo etário. Recentemente, na Austrália, Estados Unidos e alguns países da América Latina, esta área de interesse tornou-se uma sub-especialidade, tanto para médicos quanto para profissionais de outras categorias (Silber, 1997). A especialização tornou-se necessária porque a formação básica ainda não os prepara para o atendimento específico desta clientela.

Os profissionais de saúde para atender o adolescente, além de lidar com questões biológicas, têm que ter competência também na esfera comportamental. As conseqüências do uso de drogas, das práticas sexuais desprotegidas e da violência urbana passaram a fazer parte, com freqüência, do elenco de problemas deste grupo populacional (Sells \& Blum, 1996).

Nesta perspectiva, observando o movimento internacional de interesse no adolescente e considerando este segmento populacional como de risco social, a Organização Pan-Americana da Saúde (OPAS) vem incentivando a criação de programas na América Latina (OPS, 1985). No Brasil, os primeiros passos na atenção à saúde do adolescente foram dados há mais de vinte anos com a criação de serviços pioneiros no Rio de Janeiro e em São Paulo (Castells \& Silber, 1998). Com o apoio da OPAS, a partir de 1989 o Programa de Saúde do Adolescente (PROSAD) foi implantado pelo Ministério da Saúde, tornando-se um programa nacional com normas e prioridades definidas.

O PROSAD do Município do Rio de Janeiro teve suas bases programáticas publicadas em 1986 pela Secretaria Municipal de Saúde (SMSRJ ) - portanto antes da criação do Programa em nível nacional - através de uma portaria que vinculou ao Programa de Saúde Escolar os atendimentos aos adolescentes com idade até 18 anos. Atualmente o PROSAD está vinculado em instância superior à Superintendência de Saúde Coletiva da SMS-RJ e diretamente à Coordenação dos Programas de Atenção Integral de Saúde, que conta com as gerências dos Programas da Mulher, Criança, Adolescente e Idoso. Além disso, existe a Coordenação de Programas Especiais, com as gerências dos Programas de Saúde Escolar, Saúde do Trabalhador e do Projeto Rio-Criança-Cidadã. Decorridos 14 anos da implantação do PROSAD, fez-se necessária uma análise da adequação deste programa.

O objetivo deste artigo é apresentar o estudo das condições básicas das Unidades de Saúde (US) do Município do Rio de Janeiro para a prestação do atendimento integral de adolescentes de 10 a 19 anos, tendo como unidades geográficas de análise as regiões administrativas deste município.

\section{Material e métodos}

Para o estudo das condições atuais do desenvolvimento do PROSAD do Município do Rio de Janeiro realizou-se um inquérito epidemiológico na rede pública ambulatorial de saúde, utilizando-se um questionário estruturado para a coleta de dados. O instrumento foi organizado em conjuntos de itens, de acordo com o interesse dos aspectos a serem estudados. Parte do questionário foi baseada na publicação da OPAS “Aval iação de Serviços de Atendimento Ambulatorial de Adolescentes - Estimativa de Compl exi dade e Condições de Eficiência" (OPS, 1995). Os tópicos abordados foram agrupados nos seguintes conjuntos de variáveis:

a) Identificação: localização por Região Administrativa (RA); tipo e grau de complexidade da estrutura dos serviços.

b) Avaliação das condições básicas das Unidades de Saúde para prestação da atenção integral (Serrano, 1995), detalhados na Tabela 1. Como participantes do PROSAD foram consideradas todas as US que estivessem desenvolvendo qualquer atividade relativa ao atendimento ao grupo populacional em estudo, mesmo que esta participação estivesse limitada a um projeto ou a algum aspecto específico do atendimento. Quanto aos projetos procurou-se a participação nos desenvolvidos pela SMS-RJ nas US, com financiamentos de organizações nacionais e internacionais. Eram eles: Horizontes, Sinal Verde, Vista essa camisinha e Educarte.

Para avaliar as condições dos profissionais para a prestação da atenção integral perguntou-se se a equipe contava com al guns insumos, como balança, tabelas, disponibilidade de normas técnicas, etc. Quanto à possibilidade de atendimento qualificado, inquiriu-se sobre treinamento profissional e a forma como os adolescentes eram recebidos e encaminhados em caso de emergência.

Devida a importância das atividades de promoção de saúde e de prevenção aos agravos buscou-se informações se o PROSAD estava 
Distribuição dos escores atribuídos de acordo com os critérios para cada variável.

\begin{tabular}{|c|c|c|c|c|}
\hline \multirow[t]{2}{*}{ Itens de avaliação } & \multicolumn{4}{|c|}{ Escores atribuídos } \\
\hline & 3 & 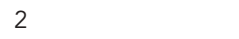 & 1 & 0 \\
\hline $\begin{array}{l}\text { 1. Participação no PROSAD* } \\
\text { Participa em alguma atividade }\end{array}$ & - & - & Sim & Não \\
\hline $\begin{array}{l}\text { 2. Participação em projetos } \\
\text { Horizontes } \\
\text { Sinal verde } \\
\text { Vista essa camisinha } \\
\text { Educarte }\end{array}$ & Todos projetos & 2 projetos & 1 projeto & Nenhum \\
\hline $\begin{array}{l}\text { 3. Profissionais capacitados } \\
\text { Médicos } \\
\text { Enfermeiros } \\
\text { Assistentes sociais } \\
\text { Psicólogos }\end{array}$ & Todos capacitados & Pelo menos 2 & Pelo menos 1 & Nenhum \\
\hline $\begin{array}{l}\text { 4. Reuniões de equipe } \\
\text { Uma vez por mês }\end{array}$ & - & - & Sim & Não \\
\hline $\begin{array}{l}\text { 5. Espaço físico disponível } \\
\text { Consultório } \\
\text { Sala para atividade de grupo }\end{array}$ & - & Ambos & Pelo menos 1 & Nenhum \\
\hline $\begin{array}{l}\text { 6. Insumos básicos } \\
\text { Balança } \\
\text { Antropômetro } \\
\text { Aparelho de pressão } \\
\text { Tabelas de peso e altura } \\
\text { Estágios de tanner } \\
\text { Ficha clínica }\end{array}$ & Todos insumos & Pelo menos 2 & Pelo menos 1 & Nenhum \\
\hline $\begin{array}{l}\text { 7. Porta de entrada na US** } \\
\text { Profissional de saúde } \\
\text { Profissional administrativo treinado }\end{array}$ & - & Ambos & $\begin{array}{l}\text { Profissional } \\
\text { administrativo treinado }\end{array}$ & Outro \\
\hline $\begin{array}{l}\text { 8. Avalia a emergência/US } \\
\text { Médico ou Enfermeiro } \\
\text { Assistente social }\end{array}$ & Médico ou Enfermeiro & Assistente social & - & Outro \\
\hline $\begin{array}{l}\text { 9. Normas por escrito } \\
\text { Equipe conhece sempre }\end{array}$ & - & - & Sim & Não \\
\hline 10. Retorno garantido & - & - & Sim & Não \\
\hline $\begin{array}{l}\text { 11. Promoção de saúde } \\
\text { Existe promoção de saúde } \\
\text { Nas escolas } \\
\text { Material educativo - sempre } \\
\text { Equipamentos disponíveis - sempre }\end{array}$ & Todos & $\begin{array}{l}\text { Atividades sem } \\
\text { material educativo }\end{array}$ & Existem atividades & Não \\
\hline $\begin{array}{l}\text { 12. Saúde reprodutiva } \\
\text { Existe atividades de saúde reprodutiva } \\
\text { Contraceptivos disponíveis sempre } \\
\text { Consulta da mãe no dia da consulta do filho } \\
\text { Atenção ao pai }\end{array}$ & Todos & - & Existem atividades & Não \\
\hline 13. Usuários de drogas & - & Ambos & $\begin{array}{l}\text { Existe atenção } \\
\text { Especial }\end{array}$ & Não \\
\hline $\begin{array}{l}\text { Existe atenção especial ao usuário de drogas } \\
\text { Atendimento à família }\end{array}$ & & & & \\
\hline $\begin{array}{l}\text { 14. Atenção à vítima de violência } \\
\text { Existe atenção especial à vítima de violência }\end{array}$ & - & - & Sim & Não \\
\hline $\begin{array}{l}\text { 15. Meninos e meninas de rua } \\
\text { Prestam atendimento }\end{array}$ & - & - & Sim & Não \\
\hline
\end{tabular}

* PROSAD = Programa de Saúde do Adolescente

** US = Unidade de Saúde 
priorizando essas atividades. Os demais itens referiram-se aos problemas mais relevantes para a população adolescente do Município do Rio de Janeiro e se as US com o PROSAD implantado estavam atuando nestes aspectos: saúde reprodutiva; usuários de drogas; vítimas de violência e meninos e meninas de rua.

Para avaliar as condições das equipes de saúde de cada US para prestar atendimento qualificado, criou-se uma pontuação - que variou de zero a três - baseada em graus de importância dos itens estudados, a partir de critérios empíricos ditados pela experiência profissional dos pesquisadores (Tabela 1). Desta forma, agrupando-se as pontuações obtidas foi possível dividir as US nas seguintes categorias: precárias, nas que os profissionais tinham menos condições de prestar atenção qualificada aos usuários; regulares: em condições um pouco melhores; boas étimas: consideradas as que os profissionais tinham melhores condições para atender a clientela. O máximo de pontuação seria de trinta.

Primeiramente, efetuou-se um pré-teste no instrumento de pesquisa, em um serviço universitário de atenção ao adolescente, procedendo-se uma reformulação dos quesitos mal compreendidos. A seguir, foram entrevistados os responsáveis pelo PROSAD, ou os coordenadores das US nas unidades sem PROSAD implantado. A análise estatística foi realizada através do programa SPSS (SPSS Incorporation, 1994).

c) Análise espacial: levando-se em conta as 26 regiões administrativas que constituíam o Município do Rio de Janeiro em 1991, foram realizados três mapas temáticos com indicadores relacionados à saúde do adolescente tendo como unidade geográfica de análise a RA. Além dos aspectos selecionados da saúde do adolescente, descritos abaixo, uma segunda camada foi sobreposta com a distribuição das US estratificadas segundo os escores que sintetizavam as condições básicas para o atendimento de adolescentes, de acordo com os critérios descritos na Tabela 1. Os mapas utilizados foram obtidos do projeto Sistema de Informação Geográfica, Fundação Oswaldo Cruz - SIG/FIOCRUZ (Pina, 1998).

O primeiro mapa temático estabelece a distribuição espacial da densidade demográfica da população adolescente, calculada pelo número de indivíduos na faixa etária de 10 a 19 anos, em 1991 (IBGE, 1994), dividido pela área (em $\mathrm{km}^{2}$ ) de cada RA.

O segundo mapa refere-se ao estudo espacial da taxa média de mortalidade por armas defogo, no período de 1991-1995, entre adoles- centes do sexo masculino (10 a 19 anos), residentes no Município do Rio de Janeiro. As populações foram estimadas através dos dados do Censo Demográfico de 1991 (IBGE, 1994) e da Contagem da População de 1996 (IBGE, 1997). Para construir a categoria de óbitos por armas de fogo, foram considerados todos os óbitos por causas externas provocados por armas de fogo classificados nas seguintes rubricas do Código Internacional de Doenças - CID9 (OMS, 1985): “homicídio com armas de fogo" (CID 965) e "Iesão por armas de fogo da qual se ignora se acidental ou intencional mente infligida" (CID 985).

O terceiro mapa descreve a distribuição das taxas de fecundidade em adolescentes de 10 a 19 anos, calculadas pelo número de nascidos vivos (NV) entre mulheres de 10 a 19 anos, no período de 1995-1997 (Sistema de Informação sobre Nascidos Vivos - dados cedidos pela Secretaria Municipal de Saúde), dividido pelo total de mulheres nesta faixa etária (IBGE, 1997).

\section{Resultados}

A rede de saúde do Município do Rio de Janeiro compreende 78 unidades (US). Infelizmente oito US não participaram do estudo por motivos variados: um hospital estava com um grave problema institucional e a diretoria não permitiu a entrada dos pesquisadores; nas outras, em diferentes localizações, os coordenadores não conseguiram agendar um horário com os pesquisadores, apesar da insistência dos mesmos. Dado o objetivo do estudo, na análise dos resultados optou-se pela exclusão destas US.

Das setenta US visitadas por ocasião da pesquisa, 49 participavam do PROSAD. Dentre as 49 US que desenvolviam atividades do PROSAD, 43 eram de nível primário, seis de nível secundário e quatro foram classificadas com os dois níveis de atenção. Quanto ao envolvimento em projetos especiais, 46 US participavam. Destas, $30 \%$ colaboravam com o Projeto Sinal Verde, $50 \%$ com o Educarte e $93 \%$ com o Vista essa camisinha.

Entre as 21 US que não participavam do PROSAD, 57\% eram de nível primário e 33\% de nível secundário, sendo que al gumas US não se auto-classificaram por níveis de atenção. Quanto aos principais motivos de não terem implantado o PROSAD, os responsáveis por 10 US (48\%) alegaram falta de treinamento técnico da equipe, de membros da equipe interdisciplinar e de demanda interessada. A falta de tempo dos profissionais e a demora no atendimento aos adolescentes em virtude da abrangência de 
assuntos a serem abordados, foram outros motivos citados.

No que diz respeito à pergunta do que seria necessário para implantar o PROSAD, mais da metade dos entrevistados referiram treinamento técnico. Os demais al egaram a necessidade de parcerias com organizações governamentais e não governamentais (ONG), ter adolescentes interessados, além de apoio da direção de suas unidades.

Em quase todas as RA existia alguma US que participava do PROSAD. Foram exceções as seguintes regiões administrativas: Portuária (RA-1), Rio Comprido (RA-3), Paquetá (RA-21) e Pavuna (RA-25), como mostra a Figura 1.

Na Tabela 2, pode-se apreciar a distribuição das US participantes do PROSAD segundo equipe interdisciplinar, categoria profissional, capacitação e disponibilidade para o atendimento no PROSAD. Cabe ressaltar que as categorias profissionais que estavam mais disponíveis e que foram capacitadas em maior número eram as de médicos e de enfermeiros. Seguiram-se os psicólogos e assistentes sociais, en-

Figura 1

Distribuição geográfica das Unidades de Saúde por adequação das condições de atendimento

e por densidade demográfica da população de 10-19 anos por quilômetro quadrado.

Município do Rio de Janeiro por Região Administrativa, 1996.

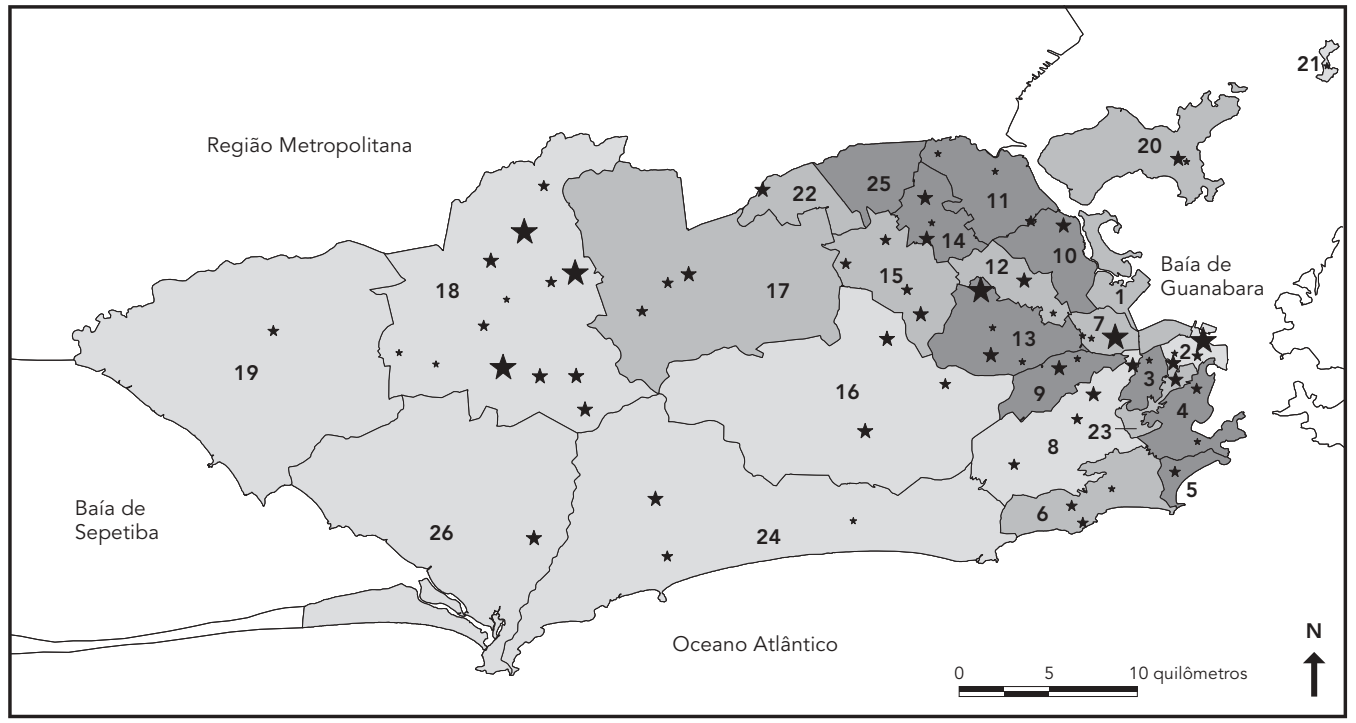

Distribuição das US por Região Administrativa

$\star \quad$ sem PROSAD

$\star$ precária

$\star$ regular

t boa
Densidade demográfica da população de adolescentes 10-19 anos por km²

menor que 801

801 a 2.001

2.002 e mais

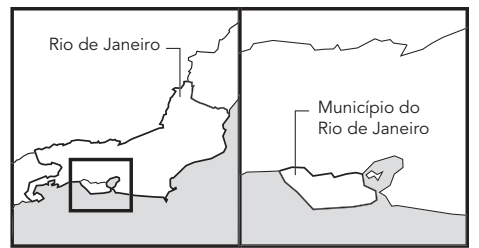

$\begin{array}{lll}\text { 1 - Portuária } & 10 \text { - Ramos } & 19 \text { - Santa Cruz } \\ \text { 2 - Centro } & 11 \text { - Penha } & 20 \text { - Ilha do Governador } \\ \text { 3 - Rio Comprido } & 12 \text { - Inhaúma } & 21 \text { - Ilha de Paquetá } \\ \text { 4 - Botafogo } & 13 \text { - Méier } & 22 \text { - Anchieta } \\ \text { 5 - Copacabana } & 14 \text { - Irajá } & 23 \text { - Santa Tereza } \\ \text { 6- Lagoa } & 15 \text { - Madureira } & 24 \text { - Barra da Tijuca } \\ \text { 7 - São Cristóvão } & 16 \text { - Jacarepaguá } & 25 \text { - Pavuna } \\ \text { 8- Tijuca } & 17 \text { - Bangu } & 26 \text { - Guaratiba } \\ \text { 9-Vila Isabel } & 18 \text { - Campo Grande }\end{array}$

Fonte: Digitalização dos mapas de Região Administrativa - Sistema de Informação Geográfica, Fundação Oswaldo Cruz. Dados tabulares: Sistema de Informação sobre Mortalidade, Ministério da Saúde. 
Equipe multidisciplinar, capacitação profissional e disponibilidade para o atendimento no Programa de Saúde do Adolescente (PROSAD) do Município do Rio de Janeiro.

\begin{tabular}{lcccc}
\hline Categorias profissionais & $\begin{array}{c}\text { Número de US com PROSAD } \\
\text { e capacitação profissional } \\
\text { Sim }\end{array}$ & Não & \multicolumn{2}{c}{ Número de US com PROSAD } \\
& & 5 & $\begin{array}{c}\text { Com Profissionais } \\
\text { disponíveis }\end{array}$ & $\begin{array}{c}\text { Sem Profissionais } \\
\text { disponíveis }\end{array}$ \\
\hline Médicos & 21 & $56(53 \%)$ & $23(47 \%)$ \\
Enfermeiros & 21 & 3 & $24(49 \%)$ & $25(51 \%)$ \\
Psicólogos & 14 & 2 & $16(33 \%)$ & $33(67 \%)$ \\
Assistentes sociais & 16 & 3 & $19(39 \%)$ & $30(61 \%)$ \\
Odontólogos & 6 & 4 & $10(20 \%)$ & $39(80 \%)$ \\
Terapeuta ocupacional & 3 & 1 & $4(8 \%)$ & $45(92 \%)$ \\
Psicopedagogos & 3 & 1 & $10(20 \%)$ & $45(92 \%)$ \\
Auxiliar de enfermagem & 3 & 7 & $5(10 \%)$ & $39(80 \%)$ \\
Adolescentes participantes & 0 & 5 & & $44(90 \%)$
\end{tabular}

US = Unidade de Saúde

quanto os demais profissionais participavam do PROSAD em número bem reduzido. Verificou-se que somente cinco US contavam com o adolescente integrando as equipes de forma rotineira, sendo que nenhum deles havia recebido capacitação.

$\mathrm{Na}$ Tabela 3 encontram-se as unidades com PROSAD distribuídas segundo os itens escolhidos para obtenção do índice que sintetiza as condições básicas. Destacam-se os seguintes resultados: $22,4 \%$ participavam em todos os projetos; $28,6 \%$ tinham a equipe mínima capacitada; em $28,6 \%$ os profissionais reuniam-se uma vez por mês; 63,3 \% contavam com espaço físico disponível; $16,3 \%$ contavam com todos os insumos básicos considerados fundamentais para a prestação da atenção integral, ressal ta-se que das 49 US, o antropômetro, ou seja equipamento para medir a altura do adolescente, estava presente em somente 26 US (53\%) e a tabela de desenvolvimento, chamada estágios de Tanner, estava disponível em trinta US (61\%); em 40,8\% a porta de entrada era avaliada por profissional de saúde ou administrativo treinado; $51 \%$ médicos ou enfermeiros avaliavam os casos de emergência; $32,7 \%$ tinham normas escritas - que estavam disponíveis - e a equipe as conhecia e utilizava; em $77,6 \%$ os adolescentes atendidos tinham retorno garantido; $18,4 \%$ faziam promoção de saúde; $6,1 \%$ tinham todos os critérios para prestarem atenção adequada quanto à saúde reprodutiva; $2 \%$ atendiam de forma especial usuário de drogas; $22,4 \%$ tinham atendimento especial a vítimas de violência e $32,7 \%$ atendiam meninos e meninas de rua.
Em relação ao índice que sintetizava as condições básicas, chamou a atenção que somente uma US tivesse alcançado a pontuação de 22, para um índice máximo de trinta pontos. Foi verificada a seguinte distribuição: $12 \%$ boas, com índices entre 20 e 22; 45\% regulares, com índices entre 15 e 19; 43\% precárias, com índices menores que 15. As demais 21 US, sem o PROSAD implantado, foram consideradas de índice zero.

Na Figura 1, observa-se que nas áreas de grande concentração populacional, localizada na Pavuna, Penha e Rio Comprido, encontravase o maior número de US que não desenvolvia atividades do PROSAD. Destaca-se também que na RA Campo Grande, que apresentava baixa densidade populacional, contava com elevado número de US em diferentes níveis de condições de atendimento do PROSAD.

Observa-se na Figura 2 que as taxas de mortalidade delimitavam três áreas distintas no município: (1) a zona portuária e redondezas da Baía de Guanabara que apresentaram os maiores índices de mortalidade por armas de fogo, no patamar a partir de 70 óbitos por 100.000 habitantes; (2) a região metropolitana com índices intermediários, de 40 a 69 óbitos por 100.000 habitantes, e (3) a zona litorânea que apresentava as taxas mais baixas, de zero a 39 óbitos por 100.000 habitantes.

Na região metropolitana, três RA registraram taxas de fecundidade com patamares elevados (Figura 3). As RA com menores taxas de fecundidade eram as próximas à orla marítima. A distribuição das US em condições adequadas, isto é, boas ou regulares, não estavam es- 
trategicamente distribuídas de acordo com esta demanda constatada. Encontrou-se uma concentração de US com diferentes pontuações nas condições de prestação de serviço aos adolescentes na RA Campo Grande e uma escassez na RA Santa Cruz, ambas apresentando elevados índices de fecundidade em adolescentes.

Fazendo um paralelo com a disponibilidade das condições das US para a prestação de serviços para a população adolescente, observou-se que, nas redondezas da zona portuária com as taxas de mortalidade mais elevadas e que apresentaram el evadas taxas de fecundidade em adolescentes, somente duas US foram consideradas em boas condições.

\section{Discussão}

A rede pública de saúde que participa do PROSAD é diversificada. Conta com serviços de alta complexidade (por exemplo: hospitais), bem como serviços com poucos recursos para prestar atendimento à população (por exemplo: Unidades de Atendimento Clínico Primário de Saúde - UACPS). Este fato deve ter contribuído, em parte, por existir na mesma rede de serviços, grande variedade de condições de atendimento nas US, classificadas de boas a precárias. Causou estranheza, entretanto, a presença de um número significativo de US em que os profissionais não tinham como proposta a participação no PROSAD. Esta constatação merece uma reflexão. Por que muitos profissionais dos serviços de saúde não estão comprometidos com o desenvolvimento de um programa que está calcado na abrangência de ações e que apresenta várias interfaces de trabalho interdisciplinar?

Alguns profissionais de saú de alegam falta de motivação para se dedicar à população adolescente, baseando-se, às vezes, nos dados epidemiológicos que apontam baixas taxas de morbidade. Não obstante, vale ressaltar que durante a adolescência o indivíduo passa por um período de rápidas mudanças tanto biológicas quanto psicossociais que propiciam comportamentos ambivalentes, ora infantis, ora maduros (Aberastury, 1971). Por isso, até chegar à fase adulta ele tem que tomar decisões, na maioria das situações sem o devido preparo, que podem implicar em riscos desnecessários (I gra \& Irwin, 1996). Motivados por exemplos de comportamento na família, pressão de grupo, auto-afirmação na comunidade, ou mesmo por dificuldades na esfera emocional, pode ocorrer a formação de hábitos pouco saudáveis, principalmente os ligados ao consumo de
Tabela 3

Distribuição (\%) das 49 Unidades de Saúde com PROSAD segundo os escores atribuídos às condições básicas para a prestação da atenção integral ao adolescente no Município do Rio de Janeiro, 1999.

\begin{tabular}{lcccc}
\hline Condiçães Básicas & \multicolumn{5}{c}{ Escores Atribuídos } \\
& 3 & 2 & 1 & 0 \\
\hline Participação em Projetos & 22,4 & 32,7 & 34,7 & 10,2 \\
Profissionais capacitados & 28,6 & 30,6 & 32,7 & 8,2 \\
Reuniões de equipe & - & - & 28,6 & 71,4 \\
Espaço físico disponível & - & 63,3 & 18,4 & 18,4 \\
Insumos básicos & 16,3 & 49,0 & 12,2 & 22,4 \\
Porta de entrada na US & - & 40,8 & 30,6 & 28,6 \\
Avalia emergência na US & 51,0 & 8,2 & - & 40,8 \\
Normas por escrito & - & - & 32,7 & 67,3 \\
Retorno garantido & - & - & 77,6 & 22,4 \\
Promoção de Saúde & 18,4 & 67,3 & 8,2 & 6,1 \\
Saúde Reprodutiva & 6,1 & 57,1 & 4,1 & 32,7 \\
Usuários de drogas & - & - & 2,0 & 98,0 \\
Atenção à vítima de violência & - & - & 22,4 & 77,6 \\
Meninos e meninas de rua & - & - & 32,7 & 67,3
\end{tabular}

PROSAD = Programa de Saúde do Adolescente; US = Unidade de Saúde * Melhores escores: 3

alimentos inadequados, experimentação de bebidas alcoólicas, fumo e drogas, entre outros. $\mathrm{O}$ importante é que esses hábitos podem originar enfermidades na idade adulta que poderiam ser evitadas com medidas preventivas.

Para que o sujeito se comprometa com a própria saúde e de seus pares torna-se necessário convencê-lo dos determinantes de seus problemas (Cerqueira, 1996). Cabe aos profissionais de saúde incentivar uma conduta responsável nos jovens, prover informações importantes e corretas sobre riscos de saúde, além de identificar os que estão em situações de risco para oferecer ajuda adequada e em tempo hábil (Di Clemente et al., 1996; Klein, 2000).

Maddaleno et al. (1992) apontam que a atenção à saúde do adolescente, pela sua própria natureza, requer o trabalho de profissionais de diferentes disciplinas. Não obstante, no Município do Rio de Janeiro, para o desenvolvimento do PROSAD, poucas US contavam com equipes compostas por médicos, enfermeiros, psicólogos e assistentes sociais, muitos dos quais sem capacitação para atenção ao adolescente. Além disso, as reuniões multidisciplinares de rotina, que poderiam ampliar as possibilidades da prestação da atenção integral e da resolução dos problemas, constituíam-se eventos raros. Outro aspecto que chamou a atenção foi a pouca participação de jovens nas equipes de saúde. Cannon (1999) enfatiza que os programas poderiam 
Distribuição geográfica das Unidades de Saúde (US) por adequação das condições de atendimento e por taxa de mortalidade por armas de fogo (por 100.000 habitantes) entre adolescentes de 10 a 19 anos. Município do Rio de Janeiro, 1991-1995.

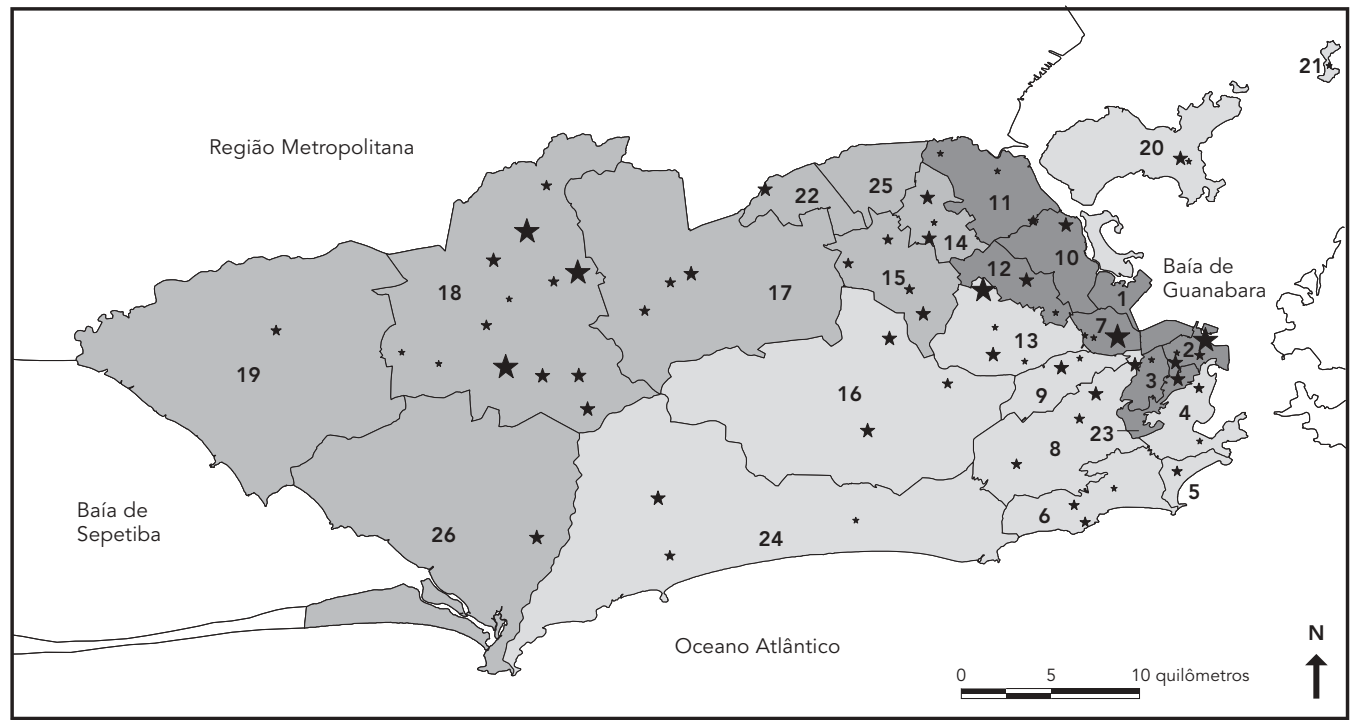

$$
\begin{aligned}
& \text { Distribuição das US Taxa de mortalidade de adolescentes } \\
& \text { por Região Administrativa do sexo masculino por } 100.000 \text { habitantes } \\
& \star \text { sem PROSAD } \square \text { a } 39 \\
& \text { ^ precária } 40 \text { a } 69 \\
& \star \text { regular } \\
& \text { 今 boa } \\
& 70 \text { e mais }
\end{aligned}
$$

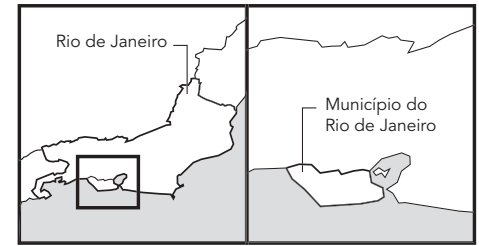

Fonte: Digitalização dos mapas de Região Administrativa - Sistema de Informação Geográfica, Fundação Oswaldo Cruz. Dados tabulares: Sistema de Informação sobre Mortalidade, Ministério da Saúde.

PROSAD = Programa de Saúde do Adolescente; NV = nascidos vivos.

beneficiar-se da presença dos jovens, para o meIhor conhecimento de suas necessidades, implementação e avaliação das atividades.

Estudando se os profissionais de saúde tinham condições de prestar atendimento integral à população adolescente do município, correlacionou-se a rede básica, distribuída espacialmente segundo critérios pré-estabelecidos, com os mais importantes problemas deste grupo etário, ou seja: a mortalidade por armas de fogo para o sexo masculino e as taxas de fecundidade para o sexo feminino.
No que diz respeito à mortalidade por armas de fogo de adolescentes do sexo masculino, observou-se que a zona portuária e adjacências apresentaram os maiores índices, denunciando a existência neste município de áreas mais problemáticas quanto ao impacto da violência. No entanto, observou-se que poucas US nessas áreas estavam disponíveis ou em condições de prestarem atendimento especializado aos adolescentes vítimas de violência. Nesta perspectiva, Blum (1987) e Flisher (1992) enfatizam a grande vulnerabilidade dos ado- 
Figura 3

Distribuição geográfica das Unidades de Saúde (US) por adequação das condições de atendimento e por taxa de fecundidade entre adolescentes de 10 a 19 anos (por 1.000 nascidos vivos).

Município do Rio de Janeiro, 1995-1997.

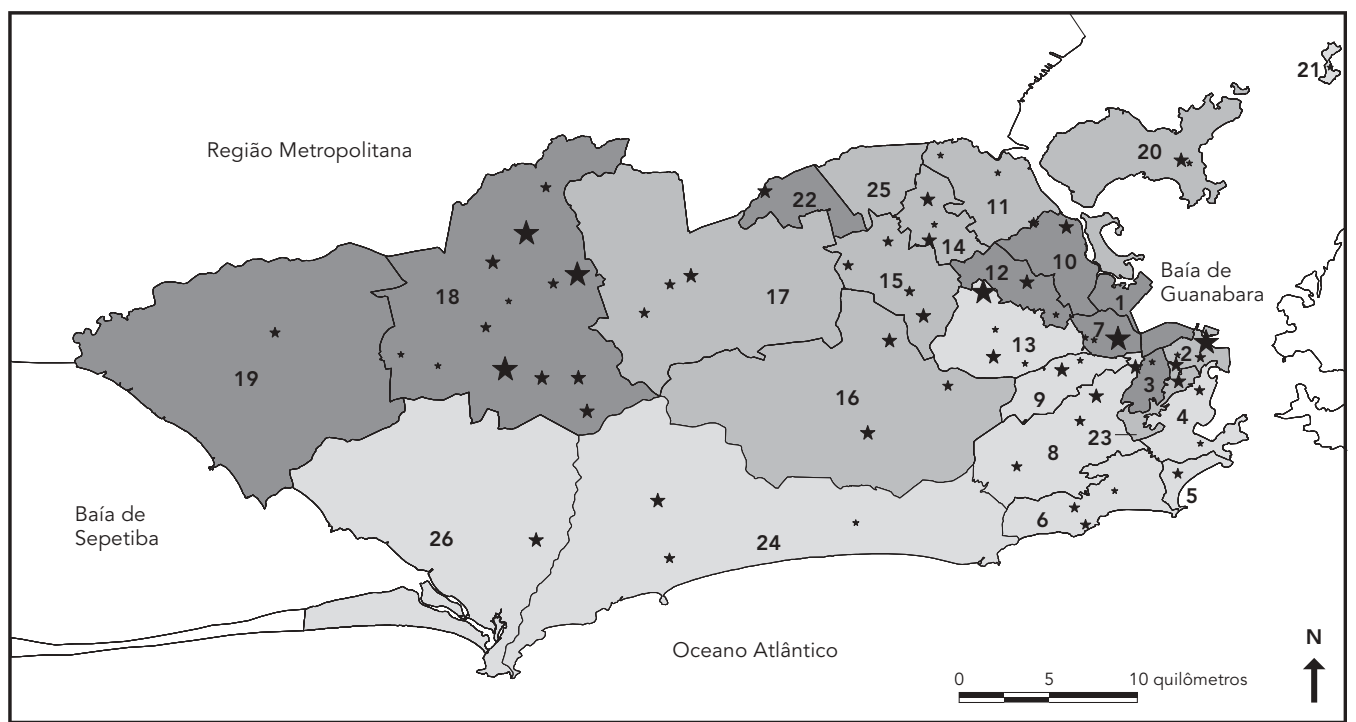
Distribuição das US
por Região Administrativa
Taxa de fecundidade
*
(/1000 NV)
$\star$ precária
$<32,0$
$\star$ regular
32,1 a 45,0
45,1 e mais
个boa
1 - Portuária
2 - Centro
3 - Rio Comprido
4 - Botafogo
5 - Copacabana
6 - Lagoa
7 - São Cristóvão
8 - Tijuca
9 - Vila Isabel

$$
\begin{aligned}
& 10 \text { - Ramos } \\
& 11 \text { - Penha } \\
& 12 \text { - Inhaúma } \\
& 13 \text { - Méier } \\
& 14 \text { - Irajá } \\
& 15 \text { - Madureira } \\
& 16 \text { - Jacarepaguá } \\
& 17 \text { - Bangu }
\end{aligned}
$$
18 - Campo Grande

\author{
19 - Santa Cruz \\ 20 - Ilha do Governador \\ 21 - llha de Paquetá \\ 22 - Anchieta \\ 23 - Santa Tereza \\ 25 - Pavuna \\ 26 - Guaratiba
}

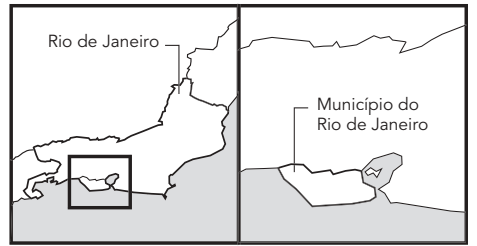

Fonte: Digitalização dos mapas de Região Administrativa - Sistema de Informação Geográfica, Fundação Oswaldo Cruz. Dados tabulares: Sistema de Informação sobre Mortalidade, Ministério da Saúde.

PROSAD = Programa de Saúde do Adolescente; NV = nascidos vivos.

lescentes do sexo masculino e propõem uma melhor atenção de saúde e das condições ambientais. Stringham \& Weitzman (1988) apontam a importância de que, na consulta de rotina de adolescentes, sejam incorporadas perguntas na história clínica quanto ao envolvimento com a violência e que sejam traçadas condutas de aconselhamento e prevenção. Em que pese o fato da rede básica de saúde não poder atuar sozinha na questão da mortalidade por armas de fogo, os profissionais que prestassem atendimento aos adolescentes com in- dícios ou suspeita de conviverem com situações de conflitos intrafamiliares ou sociais, poderiam buscar alternativas viáveis de combate à violência.

No que diz respeito à população feminina deste município, observou-se áreas com maiores índices de fecundidade, algumas delas apresentaram também taxas elevadas de mortalidade por armas de fogo em adolescentes do sexo masculino, demonstrando a multiplicidade de problemas em bairros específicos. Neste sentido, estudo realizado na Escócia compro- 
vou que as elevadas taxas de fecundidade em adolescentes aconteciam nas áreas de piores condições sócio-econômicas daquele país (McLoone \& Boddy, 1994). Da mesma forma, Stern (1997) ressalta que no M éxico os setores mais pobres apresentam uma tendência de crescimento das taxas de fecundidade na adolescência, independente da idade da primeira gravidez. Estas ponderações mostram que também, para atuar neste problema, os serviços de saúde terão que ir além do atendimento clínico, buscando parcerias com os outros setores, para obter melhor efetividade.

Embora os problemas detectados sejam decorrentes de uma série de fatores, primariamente de natureza social, e as possibilidades de prevenção através da rede de serviços de saúde sejam limitadas, o estudo sobre as condições da assistência à saúde torna-se relevante na medida em que indica deficiências que impedem os profissionais de saúde prestarem atenção de qualidade. A atenção médica vem sendo alvo da preocupação de autores voltados para os estudos sobre distribuição, acesso e eqüidade na prestação dos serviços de saúde (Travassos, 1992; White et al., 1961).

Donabedian (1982), sistematizando a avaliação da qualidade da atenção, propõe que o estudo da estrutura dos serviços de saúde deve abarcar: grau de qualificação dos recursos humanos, área física, recursos financeiros disponíveis, equipamentos em número e distribuição. Enfatiza que a estrutura é relevante na qualidade da atenção, pois aumenta ou diminui a probabilidade da boa atuação do profissional. Em nosso estudo fica evidente que as áreas de maior necessidade dispunham de poucos serviços em condições adequadas de prestar atendimento. Como obter impacto na saúde do adolescente se as US da rede pública encontram-se em condições precárias e estrate- gicamente mal distribuídas para a prestação do atendimento integral a este grupo populacional?

Na orientação de políticas públicas, Szwarcwald \& Castilho (1998) sugerem a utilização do estudo da distribuição da densidade demográfica e problemas epidemiológicos por área geográfica. Visando um impacto positivo na saúde da população adolescente do município pelo programa, algumas Regiões Administrativas merecem atenção especial por apresentarem maior concentração de problemas. Recomendase, principalmente nessas regiões, a adequação da estrutura dos serviços aos objetivos propostos pelo PROSAD, a capacitação dos profissionais de saúde em atividades de promoção de saúde, a criação de mecanismos de integração em nível local dos vários setores da sociedade como escola, saúde e comunidade, bem como, a participação dos adolescentes no desenho e avaliação dos programas a eles destinados.

O presente trabalho contou com a cooperação do gerente do PROSAD e dos profissionais que integram as equipes, assim mesmo, alguns aspectos não foram estudados por dificuldades diversas. Dentre elas, a falta de informatização de algumas unidades, o que inviabilizou a obtenção de dados como produtividade e demanda atendida. Embora a pesquisa tenha atingido cerca de $90 \%$ das US da rede, outra limitação foi quanto à impossibilidade de realização das entrevistas em todo o universo.

Conclui-se que a adequação do PROSAD às necessidades de saúde dos adolescentes pressupõe a melhoria da qualidade das condições de trabalho e da capacitação dos profissionais de saúde. Além disso, os responsáveis pela implantação dos programas assistenciais da rede municipal devem levar em conta os aspectos geográficos de concentração populacional deste grupo etário e seus principais agravos de saúde.

\section{Referências}

ABERASTURY, A., 1971. Adolescencia. Buenos Aires: Ediciones Kargieman.

BLUM, R., 1987. Contemporary threats to adolescent health in the United States. JAMA, 257:390-395.

CANNON, L. R. C., 1999. Saúdee Desenvolvimento da Juventude Brasileira: Construindo uma Agenda Nacional. Brasília: Secretaria de Políticas de Saúde, Ministério da Saúde.

CASTELLS, P. \& SILBER, T., 1998. La asistencia a adolescentes. In. Guía Práctica de la Salud y Psicología del Adolescente (P. Castells \& T. Silber, org.), pp. 33-44, México, DF: Editorial Planeta Mexicana. 
CERQUEIRA, M. T., 1996. Promoción de la salud: evolución y nuevos rumbos. Boletin dela Oficina Sanitaria Panamericana, 120:342-47.

DI CLEMENTE, R. J.; PORTON, L. E. \& HANSEN, W. B., 1996. New directions for adolescent risk prevention research and health promotion research and interventions. In: Handbook of Adolescent Health Risk Behavior - Issues in Clinical Child Psychology (R. J. Di Clemente, W. B. Hansen \& L. E. Porton, ed.), pp. 413-420, New York: Plenum Press.

DONABEDIAN, A., 1980. The definition of quality and approaches to its assessment. In: Explorations in Quality Assessment and Monitoring (A. Donabedian, ed.), pp. 3-16, v. II, Ann Arbor: Health Administration Press.

FLISHER, A. J.; JOUBERT, G. \&YACH, D., 1992. Mortality from external causes in South African adolescents, 1984-1986. South African Medicine, 81: 77-80.

IBGE (Fundação Instituto Brasileiro de Geografia e Estatística), 1994. Censo Demográfico, Brasil 1991. Rio de Janeiro: IBGE.

IBGE (Fundação Instituto Brasileiro de Geografia e Estatística), 1997. Contagem da População, Brasil 1996. Rio de Janeiro: IBGE.

GATES, A. E., 1918. The work of the adolescent clinic of Stanford University Medical Scholl. Archives of Pediatrics; 35:236-243.

HALL, G. S., 1904. Adolescence: Its Psychology and its Relationship to Physiology, Anthropology, Sociology, Sex, Crime, Religion and Education. v. 2. New York: Appleton Co.

IGRA, V. \& IRWIN, C. E., 1996. Theories of adolescent risk - Taking behavior. In: Handbook of Adolescent Health Risk Behavior (R. J. Di Clemente, W. B. Hansen, L. E. Ponton, ed.), pp. 35-48, New York: Plenum Press.

KLEIN, J. D., 2000. Adolescents, health services, and access to care. Journal of Adolescent Health, 27: 293-294.

MADDALENO, M.; KORIN, D. \& SILBER, T. J., 1992. Equipo interdisciplinario en la atención de salud de los adolescentes. In: Manual de Medicina dela Adolescencia (T. Silber, M. M. Munist, M. Maddaleno \& E. N. Suárez-Ojeda, org.), pp. 31-37. Serie Paltex para Ejecutores de Programa de Salud 20. Washington, DC: Organizacion Panamericana de la Salud.

MCLOONE, P. \& BODDY, F. A., 1994. Deprivation and mortality in Scotland, 1981 and 1991. BMJ, 309: 1465-1470.
OMS (Organização Mundial da Saúde), 1985. Classificação Internacional de Doenças, Revisão 1975 (Nona Revisão), v. 1, São Paulo: Faculdade de Saúde Pública, Universidade de São Paulo. Centro da OMS para Classificação de Doenças em Português.

OPAS (Organização Pan-Americana da Saúde), 1995. Avaliação de Serviços de Atendimento Ambulatorial de Adolescentes: Estimativa de Complexidade eCondições de Eficiência. Washington, DC: OPAS.

PINA, M. F., 1998. Potencialidades dos sistemas de informações geográficas na área da saúde. In: Saúde espaço: Estudos Metodológi cos eTécnicos de Análise(A. Najar \& E. Marques, org.), pp. 125-133, Rio de Janeiro: Editora Fiocruz.

SELLS, W. \& BLUM, R., 1996. Morbidity and mortality among US adolescents: An overview of data and trends. American Journal of Public Health, 86:513519.

SERRANO, C. V., 1995. La salud integral de los adolescentes y los jóvenes: Su promoción y su cuidado. In: La Salud del Adolescente y del Joven (M. Maddaleno, M. M. Munist, C. V. Serrano, T. J. Silber, E. N. Suárez-Ojeda \& J. Yunes, org.), pp. 3-14, Publicación Científica 552. Washington DC: Organización Panamericana de la Salud.

SILBER, T. J., 1997. Medicina da adolescência - Uma nova subespecialidade da pediatria e da medicina interna na América do Norte. Adolescencia Latinoamericana, 1:11-15.

SPSS INCORPORATION, 1994. SPSS for Windows. Statistical Packagefor the Social Sciences. Release 6.1. Chicago: SPSS Inc.

STERN, C., 1997. Teenage pregnancy as a social problem: A critical perspective. Salud Pública de México, 39:137-143.

STRINGHAM, P. \& WEITZMAN, M., 1988. Violence counseling in the routine health care of adolescents. Journal of Adolescent Health, 9:389-393.

SZWARCWALD, C. L. \& CASTILHO, A. C., 1998. Mortalidade por armas de fogo no Estado do Rio de Janeiro, Brasil: Uma análise espacial. Revista Panamericana de Salud Pública, 4:161-170.

TRAVASSOS, C., 1992. Equity in the Use of Private Hospitals Contracted by a Compulsory Insurance Scheme in the City of Rio de Janeiro, Brazil, in 1986. Ph.D. Thesis. London: Department of Social Sciences and Public Administration, London School of Economics and Political Science.

WHITE, K. L.; WILLIAMS, T. F. \& GREENBERG, B. G., 1961. The ecology of medical care. New England Journal of Medicine, 265:885-892.

Recebido em 26 de dezembro de 2000

Versão final reapresentada em 6 de agosto de 2001

Aprovado em 7 de novembro de 2001 